Summary

Examples have been given of certain words which seem to be used correctly by navigators where there is any chance of ambiguity. It is suggested that, if these and other terms can be formally defined and agreed by the Institutes of Navigation, bad language will gradually die out and confusion in navigation terminology will be reduced to a minimum. A similar exercise might also be undertaken with regard to abbreviations.

It may be that the British Institute of Navigation working with other Englishspeaking Institutes of Navigation could make a start by defining certain words in the hope that they will gradually come to be used with a specific meaning. The alternative is a continuation of the present chaos whereby an official body such as the Airlines Electronics Engineering Committee can decide that 'course' and 'track' have the same meaning ${ }^{4}$ and therefore that a direction is a line.

This note is published with the kind permission of Mr. K. Fearnside, Technical Director of Smiths Aviation Division, but is not intended to represent Company views. Mr. M. W. Richey's advice and help is gratefully acknowledged.

\title{
REFERENCES
}

1 Moody, A. B. Navigation Dictionary. H.O. 220. U.S. Government Printing Office. Washington 25, D.C.

2 Smiths Aviation Division, Navigation Terminology, R.I.D. 624. Smiths Aviation Division, Bishops Cleeve, Gloucestershire, U.K.

3 Moody, A. B. 'Do you say what you mean ?' Navigation U.S.A., 4, 27.

4 Airlines Electronics Engineering Committee. Letter No. 65.1.40. 1700K Street N.W., Washington 6, D.C.

\section{The Exponential Integral Frequency Distribution}

\author{
J. B. Parker
}

I. INTRODUction. In a recent note, D. A. Lloyd 1 has obtained a formula for the frequency distribution of time dependent errors in terms of the exponential integral

$$
-E i(-z)=\int_{z}^{\infty} \frac{e^{-q}}{q} d q
$$

This note clarifies Lloyd's derivations by referring to a theoretically identical, though conceptually different, time-independent, physical model, relates this distribution to one described by Anderson 2 and concludes with a short appreciation of the role of the negative exponential distribution in navigational studies.

2. THE MODEL. We imagine a situation in which the error data are heterogeneous, consisting of a combination of several Gaussian distributions, each of 
zero mean, but with a whole range of standard deviations between the limits $L_{1}$ and $L_{2}$. The probability density function for the error $x$ is clearly

$$
h\left(x ; L_{1}, L_{2}\right)=\int_{L_{1}}^{L_{2}} f(x, \sigma) g(\sigma) d \sigma / \int_{L_{1}}^{L_{2}} g(\sigma) d \sigma
$$

where $g(\sigma)$ is the probability density function for the s.d. $\sigma$ and $f(x, \sigma)$ is the Gaussian density function

$$
f(x, \sigma)=\frac{\mathrm{I}}{\sqrt{ } 2 \pi \cdot \sigma} \exp -\frac{x^{2}}{2 \sigma^{2}}
$$

This situation could be regarded as representing a population of errors made by different navigators of varying degrees of proficiency (different $\sigma$ ) and perhaps under different operating conditions (again, different $\sigma$ ).

If now it be assumed that $\sigma$ is equally likely to lie anywhere between $L_{1}$ and $L_{2}$ then $g(\sigma)$ takes the simple form

so that, after a change of variable,

$$
g(\sigma)=\left(L_{2}-L_{1}\right)^{-1}
$$

$$
h\left(x ; L_{1}, L_{2}\right)=\frac{1}{2\left(L_{2}-L_{1}\right) \sqrt{2 \pi}}\left[E i\left(-\frac{x^{2}}{2 L_{1}^{2}}\right)-E i\left(-\frac{x^{2}}{2 L_{2}^{2}}\right)\right]-(\mathrm{I})
$$

This gives Lloyd's results when $L_{1}=0$; for, in the one-sided distribution his time average of the variance is given by

$$
\left(\sigma^{2}\right)_{1}=\frac{L_{2}^{2}}{3}\left(1-\frac{2}{\pi}\right)
$$

whilst the true variance of the distribution is given by

$$
\left(\sigma^{2}\right)_{2}=\int_{0}^{\infty} 2 h\left(x ; 0, L_{2}\right) x^{2} d x-\left\{\int_{0}^{\infty} 2 h\left(x ; 0, L_{2}\right) x d x\right\}^{2}=L_{2}^{2}\left(\frac{1}{3}-\frac{1}{2 \pi}\right)
$$

Substituting for $L_{2}$ in our formula ( $\mathrm{I}$ ) for $h\left(\mathrm{x} ; \circ, L_{2}\right)$ yields Lloyd's result at the foot of page I I 9 of his paper. His 'double-sided' result (p. I 22 ) is obtained by verifying that

$$
\left(\sigma^{2}\right)_{3}=\int_{-\infty}^{\infty} h\left(x ; \circ, L_{2}\right) x^{2} d x=L_{2}^{2} / 3
$$

The general form ( 1 ), for which the population variance is

$$
V=\left(L_{2}^{2}+L_{2} L_{1}+L_{1}^{2}\right) / 3
$$

and the average absolute error is

$$
A=\frac{1}{\sqrt{2 \pi}}\left(L_{1}+L_{2}\right)
$$

is an exact description of the error distribution we have described. It raises the speculation that populations that are known to be heterogeneous might possibly be graduated in terms of the two parameter probability density function (I) which, as we have introduced it, is an expression for errors over the whole range $(-\infty, \infty)$ - 'double sided' in Lloyd's terminology; the corresponding onesided distribution is of course $2 h\left(x ; L_{1} L_{2}\right),(0 \leqslant x \leqslant \infty)$, since the functional is symmetrical about $x=0$. 
The asymptotic expansion for the distribution function corresponding to ( $\mathrm{I}$ ), which gives the chance that the error exceeds $x$ when $x$ is large, is

where

$$
\frac{1}{L_{2}-L_{1}}\left\{Q\left(L_{2}, x\right)-Q\left(L_{1}, x\right)\right\}
$$

$$
\begin{aligned}
Q(L, x)=\frac{L}{\sqrt{2 \pi}} \exp -\frac{x^{2}}{2 L^{2}} \cdot\left[\left(\frac{L}{x}\right)^{3}-5\left(\frac{L}{x}\right)^{5}\right. & +\cdots \\
& \left.-(-)^{r}\left(\frac{L}{x}\right)^{2 r+1}\left\{2^{r} . r !-\frac{(2 r) !}{2^{r} . r !}\right\}+\cdots\right]
\end{aligned}
$$

This formula is quoted because it shows that the distribution (I) is not asymptotically negative exponential, unlike the distribution of extremes instanced by Crossley. 4

3. Discussion. For large $L_{2}$ and small $L_{1}$ the slightly more general form (I) tends to Lloyd's double-sided distribution (loc. cit., P. I 22 ) and as he remarks the distribution approximates closely to the negative exponential over a wide range of the variable $x$ (though it is not asymptotically of this form). Although Lloyd has already shown this effect graphically in a clear and unambiguous manner, it is of interest to portray the case $L_{1}=1 ; L_{2}=10$ for this corresponds to the situation illustrated by Anderson in Fig. 6 of his paper. ${ }^{2}$ The plot is shown, for the single-sided case, in Fig. 1 .

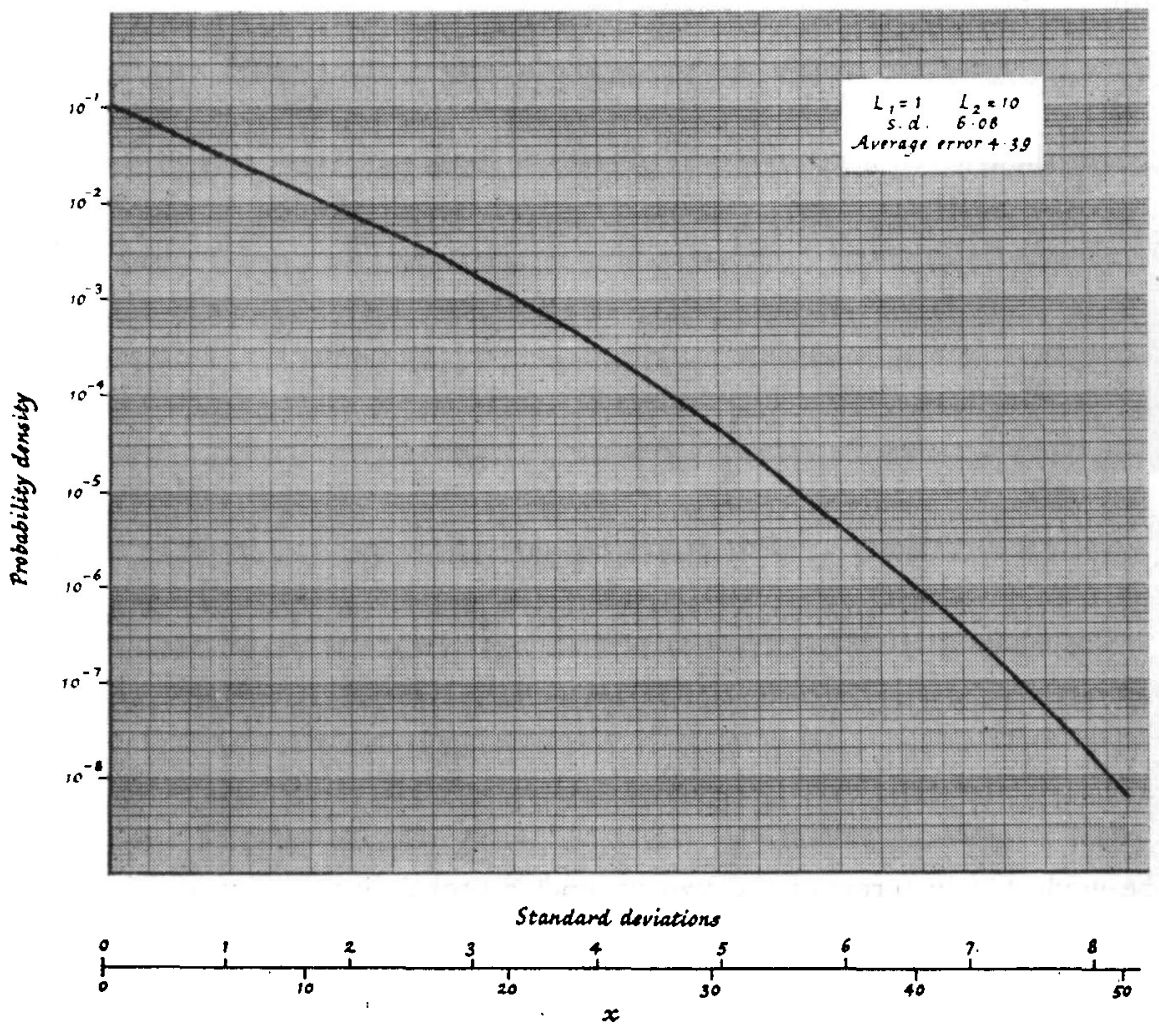

FIG. 1. The probability density function 
Although to the theoretician there may be a temptation to essay a fit of form (1) to actual operational data, in practice it does not look promising. The data obtained by the Astronomical Observations At Sea Working Party 3 are not graduated by (1), however $L_{2}$ and $L_{1}$ are chosen; these data are certainly heterogeneous and at first sight might be thought to obey the situation described at the start of the preceding paragraph. There are many possible reasons for the failure of this graduating process, such as the presence of blunders, the failure of the probability density function $g(\sigma)$ to be uniformly distributed, and the presence of systematic errors amongst part of the basic data.

It is instructive to note the recent papers 1,4 which shed light on the approximate negative exponential behaviour of errors (of large errors in particular) observed in practice in a wide number of different fields $;^{2}$ in most examples the error data have been heterogeneous. It is, however, wrong in my opinion to go to an extreme and decry the Gaussian distribution because it does not graduate data for which it is inappropriate. There is a danger that recent evidence be used to call in doubt.the validity of Gaussian methods over the whole field of navigation; this is wrong since in many-indeed, to the practical navigator, most-applications the data are not markedly heterogeneous. To take a simple example, when using probabilistic methods to determine position, 5 the navigator is concerned not with the errors all navigators made under a wide range of operational conditions, but with his own errors made under a specific set of circumstances. It seems to me that Gaussian methods are appropriate in this context. It is hoped to review the whole subject at greater length in a future article.

\section{REFEREN CES}

1 Lloyd, D. A. (I 966$)$. A probability distribution for a time-varying quantity. This Journal, 19, 119.

2 Anderson, E. W. (1965). Is the gaussian distribution normal ? This Journal, I8, 65.

3 The accuracy of astronomical observations at sea (Working Party Report). This Journal, 10, 223.

4 Crossley, A. F. (1966). On the frequency distribution of large errors. This Journal, 19, 33 .

5 Parker, J. B. (1952). Simultaneous position data in the air. This Journal, 5, 235.

\section{A Radar Display for Collision Avoidance}

$$
\text { Commander P. C. H. Clissold, R.N.R. }
$$

It is, I believe, generally agreed that if the aspect of a ship could be seen on the radar screen from a range of 4 or 5 miles down, the information provided by the radar picture would be sufficient to prevent collisions from occurring. The aspect cannot be directly observed on an ordinary $3-\mathrm{cm}$. marine radar, but I think such a set could be modified in the following way to enable the aspect to be shown. 